Voix et Images

voixetimages

\title{
L’écriture mutante dans la Québécoite de Régine Robin
}

\section{Madeleine Frédéric}

Volume 16, numéro 3 (48), printemps 1991

François Charron

URI : https://id.erudit.org/iderudit/200924ar

DOI : https://doi.org/10.7202/200924ar

Aller au sommaire du numéro

Éditeur(s)

Université du Québec à Montréal

ISSN

0318-9201 (imprimé)

1705-933X (numérique)

Découvrir la revue

Citer cet article

Frédéric, M. (1991). L'écriture mutante dans la Québécoite de Régine Robin.

Voix et Images, 16(3), 493-502. https://doi.org/10.7202/200924ar d'utilisation que vous pouvez consulter en ligne.

https://apropos.erudit.org/fr/usagers/politique-dutilisation/ 


\section{L'écriture mutante dans la Québécoite de Régine Robin}

\section{par Madeleine Frédéric, Université libre de Bruxelles}

La modernité-monde dont parle Jean Chesneaux dans son dernier ouvrage ${ }^{1}$ entraîne, parmi bien d'autres conséquences, une nouvelle relation à l'espace et au temps. Au plan spatial, la rue cesse d'être un lieu de socialité, défini entre autres par ses fonctions d'échange, de relations humaines informelles, de vie collective. Elle disparaît au profit d'axes véhiculaires où l'on ne fait plus que passer, l'équipement urbain ne visant plus qu'à accélérer la circulàtion, le mouvement, le passage ${ }^{2}$. Le temps de la modernitë, quant à lui, se contracte dans l'immédiat; l'instantané devient un véritable impératif moral, l'urgence une idéologie. La vitesse est le critère supérieur de performance pour les engins comme pour les gens, et Jean Chesneaux donne en exemple la devise du T.G.V.: Gagner du temps sur le temps ${ }^{3}$. Ce repli sur le présent affecte notre sensibilité personnelle, dégrade nos perceptions collectives. Nous perdons le sens de l'appartenance à une succession de générations enracinées dans le temps et donc se prolongeant dans le futur. La modernité, nous dit Chesneaux, est amnésiante, l'homme moderne est en état d'apesanteur historique ${ }^{4}$. Cette relation complètement modifiée à l'espace et au temps, il nous a paru intéressant de voir quelle expression elle trouvait en littérature, en particulier dans un roman récent, la Québécoite de Régine Robin ${ }^{5}$, qui y fait largement écho, aussi bien au plan des thèmes qu'à celui des techniques d'écriture.

Au plan thématique, l'ouvrage apparaît exemplaire, dans la mesure où lä figure féminine centrale est en proie à une double désorganisation spatio-temporelle: celle inhérente à la métropole moderne qu'est Montréal, mais aussi celle qui tient à sa condition d'immigrée confrontée, comme elle le dit dès les premières lignes, à la nuit noire de l'exil, à l'Histoire en morceaux (p. 45). Deux figures proches de la narratrice par leur judaïté déjà parviennent à résister à l'errance, à lutter

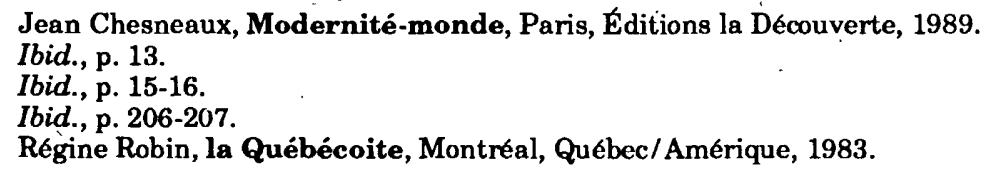


contre la modernité amnésiante: le vieil écrivain asthmatique, protagoniste du roman impossible entrepris par elle, a choisi de soumettre ses étudiants à un conditionnement intensif: D'abord de l'histoire, toujours de l'Histoire - leur enfoncer le cul dans l'Histoire. (p. 37) Quant à Mimi Yente, elle a transplanté avec elle ses racines qui tiennent à peu de choses en fait: au vieux samovar de Jitomir, dont la mention scande le roman telle une basse continue, et à la célébration du sabbat, non par religiosité, comme elle l'explique à la narratrice agacée, parce que c'est une façon de se souvenir qu'on est juif (p. 132).

Pour la narratrice, la tâche semble plus ardue. La solution est à chercher au plan de l'écriture: c'est par le biais de l'écriture qu'elle espère parvenir à appréhender la réalité complexe de la métropole moderne:

parler d'un hors-lieu; d'un non-lieu, d'une absence de lieu. Essayer de fixer, de retenir, d'arracher quelques' signes au vide. [...] faire un inventaire, un catalogue, une nomenclature. Tout consigner pour donner plus de corps à cette existence. Tes menus faits et gestes, tes rencontres, tes rendez-vous - tes itinéraires - les consonances bizarres des grands magasins [...]. Tout cela finirait bien par avoir l'épaisseur d'une vie, d'un quotidien. Serait-il possible de trouver une position dans le langage, un point d'appui, un repère fixe, un point stable; quelque chose qui ancre la parole alors qu'il n'y a qu'un tremblé du texte, une voix muette, des mots tordus? (p. 18-19)

C'est précisément ce passage qui va nous fournir un fil précieux pour entrer plus avant dans ce roman à l'écriture véritablement mutante, à l'image de la réalité métropolitaine qu'elle tente de cerner. Cette quête d'une position de langage se concrétise, en effet, de différentes façons dans l'œuvre; on s'attachera ici à l'examen de ce qui se révèle un procédé stylistique récurrent: l'énumération et l'utilisation très particulière qu'en fait l'auteure.

Il semble à l'analyse que l'on puisse regrouper en deux grands types les diverses énumérations qui parcourent l'œuvre. Un premier est formé de séries énumératives à caractérisation nulle. Simple catalogue, inventaire nu, véritable déboulé de noms, mis bout à bout, dans l'ordre où les données qu'ils désignent se sont imposées à la narratrice au hasard de la marche, d'un trajet en voiture, d'une lecture, etc. Elle se contente de les relever, le plus exhaustivement possible, mais sans chercher à les particulariser, à les individualiser. Ces séries sont abondamment représentées dans l'œuvre. Toutes ont trait à la réalité montréalaise: défilé de grands magasins (p. 18), de banques (p. 23), d'enseignes aperçues le long d'une route à Longueuil (p. 50-51), de crêperies et de bars (p. 53), de commerces bordant différentes artères: une section de la rue Sherbrooke (p. 62-63), de Saint-Denis (p. 142-143), de Victoria (p. 183), ou encore de fragments 
successifs de la Main (p. 64, 140 et 174); inventaire des stations de métro (p. 51-52), d'agences immobilières (p.' 72-73), de restaurants fast-food et d'hôtels (p. 78); relevé des Saint-Hubert Barbecue (p. 185); examen de la carte de l'Omelette Saint-Louis (p. 149-150); passage en revue d'annonces publicitaires (p. 140-141), de programmes T.V. (p. 124-128), de résultats de hockey enchaînant sur une évocation sans concession du Québec, $51^{e}$ état du drapeau américain avec une fleur de lys (p. 81-84). À travers elles, c'est la société de consommation montréalaise qui nous est livrée telle quelle, de façon brute, sans fioriture aucune. Elle ne sont pas sans rappeler les énumérations du chantre de la modernité, le poète américain Walt. Whitman, à la différence significative près qu'elles restent cantonnées au niveau de la réalité la plus quòtidienne, sinon la plus prosaïque.

Par le biais de ces relevés méthodiques, une même entreprise est visée par la narratrice: remettre bout à bout les pans de cette ville morcelée, de cette ville sans cohérence, sans unité, afin d'essayer de l'assimiler, de se l'incorporer (p. 167) enfin. Cette cohérence qu'elle cherche désespérément ne débouche parfois que sur la redondance pure et simple:

Fixer la différence de toutes ces banques-répandues dans la ville comme des mouches

Banque canadienne nationale

Banque de commerce canadienne impériale

Banque de Montréal

Banque de Nouvelle-Écosse

Banque d'Épargne

Banque du Canada

Banque fédérale de développement

Banque mercantile du Canada

Banque provinciale du Canada

La Toronto Dominion Bank

banque - banque - le pays des banques - the big bank country - the big bank power

in God we bank. (p. 22-23)

Toutefois, malgré la difficulté de l'entreprise, elle insiste sur sa volonté d'exhaustivité; la formule [n]oter toutes les différences revient tel un leitmotiv:

Noter toutes les différences. Les plaques avant et arrière des autos. Je me souviens. Montréal je t'aime. J'aime ma femme. L'emblème des Canadiens, l'emblème des Expos, ou des prénoms, des lieux: Gaspé, Rivière-du-Loup, Saint-Georges-deBeauce. Une fois aussi, par exception, Allende te recuerdo. Chile no te olvido. Le drapeau italien, le drapeau grec ou la feuille d'érable, ou la fleur de lys ou à la fois du bleu et du rouge, la feuille d'érable et le fleur de lys. 
Tout noter. Ne rien oublier. L'urgence. Tout emmagasiner, comme si tu devais te retrouver tel Robinson sur son île et ne plus rencontrer Montréal que par traces, signes, symboles, fragments sans signification, morceaux, débris, tessons hors d'usage. L'amour obsessionnel des listes, des inventaires, des archives. Historienne du rien, du fugace. Angoisse de la trace à garder. (p. 196)

Car tel est bien le sens profond de cette quete: lutter contre l'effacement des choses, tout en redonnant à cette ville sans temps ni lieu une certaine unité. C'est, en effet, une même image de la ville nordaméricaine qui se dégage des mouvements de synthèse s'amorçant ici et là au terme d'une série:

Une Amérique mal ficelée, toute juxtaposée en vrac. Comprimé. de temps et d'espace, tous les pays, toutes les Histoires, tous les peuples. Ecuménisme du pauvre, du poursuivi, de celui qui n'a pas le droit à la parole. En vrac. Éclaté. L'Amérique de toujours. Pas un pays. Des imaginaires, des nostalgies. Des remakes, des ersatz. (p. 64)

Une énumération surtout est illustrative de ce profond désarroi. Elle apparaît aux pages 152-153 et est scandée par l'interrogation angoissée [n]'y a-t-il rien d'universel ici ${ }^{6}{ }^{6}$ Avec cette mention - même négative - de l'universel, le ton à tendance à changer. Les notations plus subjectives apparaissent (cf. entre autres: Comme le ciel est loin et quel silence givre sur les branches!), les marques de l'affectivité investissent le discours. On est loin des notations sèches qui faisaient la substance des séries précédentes. Ce passage offre d'ailleurs une bonne transition vers l'examen du second type d'énumérations, qui reposent quant à elles sur une caractérisation nettement plus fournie: déterminants, adjectifs, relatives, circonstants, etc. y abondent. Elles sont réservées de manière privilégiée à l'évocation du Paris d'autrefois, qui refait surface malgré elle dans la mémoire de la narratrice:

Chevaleret. Place d'Italie avant l'éventrement du quartier. Les petits bistrots autour de la place, l'entrée d'un magasin, la rue Bobillot où la petite allait faire de la poterie le jeudi je crois. Il y a si longtemps. L'atelier était au fond d'une cour. C'était encore le Paris des artisans. Il y avait des cours intérieures, des maisons basses aux toits biscornus. C'était avant les grandes tours et les supermarchés. Au fond de la cour il y avait un marché avec de la menthe et de la marjolaine. On entendait les machines du tailleur à côté et un ferblantier un peu plus loin. La vie a passé depuis. Corvisart, Glacière. Il fallait

6 Parfois au milieu des collègues, des amis, elle serait prise d'une grande panique [...] Il y aurait encore des pays de mimosas. Peut-être! 
descendre à la Glacière, prendre la rue du même nom jusqu'au carrefour Reille, prendre la rue de la Colonie pour arriver à la place de l'Abbé Henocque. Cette place si calme, si ombragée. C'est là qu'ils ont tué P. Goldmann - les souvenirs du juif polonais né en France s'arrêtent là. UN JUIF POLONAIS ASSASSINÉ EN FRANCE. Tu te souviens de cette douleur violente quand tu a appris la nouvelle. Autour de la Place, il y a des vieilles maisonnettes bordées de jardinets. On $y$ serait bien dans cette maison. (p. 99)

Cette évocation du Paris pittoresque d'autrefois s'appuie sur des constituants nettement caractérisés: circonstants, relatives, adjectifs, énumérations intérieures concourent à cet effet; en même temps qu'elle voit se multiplier les axiologiques ${ }^{7}$ (recours à l'intensif: $S I$ calme, SI ombragée; jeu des adjectifs et des diminutifs: maisonnettes, jardinets, petits bistrots en face dé: les grandes tours, les supermarchés, etc.) - la narratrice n'hésitant pas, en outre, à intervenir directement dans son énoncé (je crois, on y serait bien). Sans prétendre dresser ici le. relevé complet des traces laissées par la narratrice dans cette évocation qui est tout sauf neutre, relevons encore au passage l'utilisation du syntagme - hautement déictique et affectif - la petite, ainsi que le recours à ce que Tesnière appelait la projection des actants: on $y$ serait bien dans cette maison ${ }^{8}$. L'intertextualité vient donner encore plus de relief au tableau. L'allusion au jardin parfumé évoquant insensiblement la chanson de Jacques Dutronc, C'était un petit jardin. Ce relief, la fin du passage l'accentuera encore, en conférant à la place de l'Abbé Hénocque une dimension historique et politique qui n'est sans doute pas la moindre raison de l'attachement qu'y porte la narratrice.

On constate, en effet, que le côté pittoresque ne fait que croître avec l'intrusion du politique dans ce Paris humain et déjà profondément convivial; c'est qu'à la socialité du quartier vient alors s'ajouter la solidarité née d'une même lutte: l'évocation du quartier de la Bastille et de sa métamorphose les jours de 14 juillet le montre bien (p. 172-173). Mais un passage surtout est particulièrement révélateur à ce propos. Il se situe aux pages 113-116: trois pages, la longueur de la série déjà a de quoi rétenir l'attention; mais en outre, il apparait dans un environnement qui semble lui aussi non innocent: occupant à peu de choses près la partie centrale du roman, il est précédé de peu par une énumération du premier type, colligeant les spécialités de chez Bens en une énumération sèche, nue (p. 111-112), du type de celles

7 Cf. à ce propos Catherine Kerbrat-Orecchioni, l'Énonciation. De la subjectvité dans le langage, Paris, Armand Colin, 1980.

8 Pour les différentes valeurs attachées à ce tour, cf. entre autres Madēeine Frédéric, la Répétition. Étude linguistique et rhétorique, Tübingen, Max Niemeywer Verlag, 1985, p. $98 \mathrm{sq}$. 
évoquées au début de cette analyse, réservées, on l'a vu, à l'évocation de la réalité montréalaise. L'ensemble est encadré par deux formules mathématiques ${ }^{9}$ qui donnent sans ambages la clef de l'ensemble:

Voici quelques-unes de nos spécialités de renommée mondiale...

èt

\section{BENS une tradition montréalaise.}

Cette dernière a de quoi faire sourire quand on songe au vaste patchwork culinaire étalé dans l'intervalle.

Dans la foulée apparaît le récit de la réussite de Bens (p. 112-113), parfaitement dans le ton du mythe américain du self-made man et dont la première lecture déjà fait ressortir plusieurs tics du langage propre aux prospectus publicitaires. Puis, tout à coup, on assiste au surgissement involontaire de la mémoire (l'évocation de cette remontée, de cette insinuation de la mémoire a d'ailleurs une certaine résonance proustienne). C'est à ce passage que je faisais allusion plus haut. Évoquant le Paris politique viscéralement lié au passé de la narratrice, il consiste en une suite de slogans et de mots d'ordre mis bout à bout. En cela, on pourrait dire qu'il n'est guère différent du catalogue des spécialités de Bens; mais c'est précisément là que réside le rôle des mouvements de synthèse encadrant la série. Par le mouvement d'ouverture qu'ils amorcent, par leur élargissement à l'ensemble de la condition humaine et leur inscription dans l'Histoire (cf. La rumeur des grandes foules, des morceaux d'Histoire ratifiée [p. 113] et coupée de son Histoire [p. 116]), ces mouvements de synthèse tendent à rapprocher le passage des séries homologiques rencontrées chez Saint-John Perse, du moins - et jy insiste - par leur objectif qui est de viser à l'universel, les moyens d'atteindre ce dernier étant radicalement différents. Si la narratrice de la Québécoite tente de le cerner par le biais de mouvements collectifs, de scènes de foules, poussant à son degré maximum la solidarité dans les luttes qui fait à ses yeux l'un des charmes du Paris d'autrefois, chez Saint-John Perse en revanche l'universel est atteint au travers de petites scènes individualisées, par le biais de constituants bénéficiant d'une large autonomie, en citant à la barre des garants très singuliers, dont le côté le plus souvent solitaire ou du moins marginal eśt encore accentué par le recours privilégié au singulier ${ }^{10}$. Dans la Québécoite, en revanche, l'individu se fond dans

9 Cf. à ce propos Madeleine Frédéric, la Répétition et ses structures dans l'ouvre de Saint-John Perse, Paris, Gallimard, 1984, p. 133 sq.

10 Pour une analyse concrète de cette technique, $c f$. Madeleine Frédéric, "Énumération, énumération homologique, énumération chaotique. Essai de caractérisation ", Stylistique, rhétorique et poétique dans les langues romanes, Publications de l'Université de Provence, vol. VIII, 1986, p. 103-117. 
la masse au niveau des mouvements de synthèse (rumeurs, grandes foules), mais surtout, il s'efface complètement derrière des mots d'ordre dans le mouvement proprement analytique de la série.

Il n'en reste pas moins que ce long passage marque un très net saut qualitatif dans la quête de l'universel poursuivie par la narratrice; ce qui ressort nettement si on le compare avec la série évoquant les spécialités de chez Bens. Dans l'úne et l'autre série, les constituants du mouvement analytique sont mis bout à bout ${ }^{11}$; mais si dans la série relative à Bens, on l'a vu, le lien entre formules synthétiques et éléments détaillants est donné immédiatement, ce lien devient plus indirect dans la série "parisienne". Il est encore aisément perceptible dans des syntagmes comme des fragments de conversations, de meetings, de réunions ou la rumeur des grandes foules, des manifestations, des mots d'ordre, des chansons, des slogans des banderoles, mais il tend à se faire beaucoup plus lâche dans la référence à l'Histoire, qui encadre la série: ce dernier lien n'est plus à proprement parler de type hypéronymique.

Il est intéressant de constater que la réminiscence involontaire de ce passé militant parisien est suivie d'une évocation des luttes politiques au Québec:

Elle se serait constituée pour elle-même des analogies, des repères, des événements avec lesquels ici elle pourrait s'identifier. Des luttes qu'elle comprendrait, un langage commun. Quelque chose de vaguement universel qui lui permettrait de ne pas sentir la démembrure. Un catalogue disparate, un vade-mecum pour exilé de gauche. Une énorme besace dans laquelle on trouverait pêle-mêle sans aucune chronologie d'abord le manifeste du F.L.Q. (p. 116)

Le texte dudit manifeste occupe les pages 116 à 122 ; l'exploration du contenu de la besace (l'expression revient comme un leitmotiv, qui scande et structure le passage) se poursuit: luttes politiques et syndicales, combat des femmes, agit-prop et tentatives de rénovation artistique sont successivement passés en revue (p. 122-124) et sera-t-on vraiment étonné d'observer qu'elles le sont non plus par le biais d'un catalogue froid et nu, mais au travers d'un discours du second type, nettement marqué par la subjectivité de l'instance narratrice ${ }^{12}$.

11 Dans l'une, l'impression de disparate nait du rejet à une nouvelle ligne de chaque constituant supplémentaire; dans l'autre, la disposition cursive atténue quelque peu cette impression.

12 Il est intéressant de comparer ce passage avec une série du premier type qui énumère, notamment, les différents partis politiques: Noter toutes les différences [...] Pénétrer l'étrangeté de ce quotidien. (p. 176-177) Les partis politiques voisinant indifferemment avec les marques de dentifrice, de savon ou de lessive... l'amalgame dit bien l'absence de cohérence de cette cité "schizophrène"; il dit aussi clairement qu'il ne faut pas confondre luttes et partis politiques. 
Ainsi donc, il apparaît que ce clivage entre séries du premier type et séries du second répondrait peutêtre moins à un clivage entre réalité montréalaise et réalité parisienne que bien plutôt à celui qui distinguerait brave modern world - pour reprendre l'expression de Chesneaux -, ville moderne privée d'âme d'une part, cité conviviale de l'autre.

C'est ce qui explique que telle série parisienne dérape soudain, lorsqu'on aborde le Paris d'aujourd'hui:

La France aussi

une colonie.

La France des petites places des vieux quartiers.

Des coins de rue aujourd'hui disparus

'de l'apéro, du pastis, et du calva sur le zinc.

Aujourd'hui

Carrefour

Mammouth écrase les prix

Europe 1 c'est naturel

Mettez un tigre dans votre moteur

La France des hauts de Belleville

Le Paris des appartements à

$285.000 \mathrm{~F}: 50 \mathrm{~m}^{2}$ charmant deux pièces

2.300.000 F magnifique appartement de $180 \mathrm{~m}^{2}$ dans l'île

Saint-Louis

$375.000 \mathrm{~F}$ beau deux-pièces dans le XIVe.

La France paumée

assassinée

avec des graffitis antisémites sur les murs du métro.

Pleure pas tout est fini

ville schizophrène

sans feu

ni lieu

Espace nomade. (p. 86)

La cité d'antan se heurte à la métropole actuelle et la série du second type qui semblait vouloir s'ébaucher (lignes 3 à 5 ), s'étrangle tout aussitôt pour faire place à des slogans publicitaires vidés d'âme, rappelant les inventaires dressés par la narratrice à Montréal. Une même dénonciation de ville schizophrène sert d'ailleurs tour à tour pour Montréal (p. 80) et Paris (p. 86).

L'allusion à Belleville prolonge la peinture haute en couleurs brossée aux pages 54 à $56^{13}$, en même temps qu'elles anticipent l'une et l'autre sur l'analyse qu'en fera Jean Chesneaux cinq ans plus tard:

13 Etais-tu moins seule dans cette foule animée du samedi soir se pressant à la Contrescarpe. [...] Les marrons chauds, les crêpes au rhum, les roudoudous et les boites de cocos. 
Cour de la Commune en 1871, la vieille commune ouvrière de Belleville dans le nord de Paris, qui fut longtemps un vrai village, avait conservé jusque dans les années 60 une forte socialité, des rapports de voisinage très vivants, une culture populaire inscrite dans la langue et les anecdotes, dans les cheminements familiers et les habitudes. Les cortèges du Front populaire $y$ furent maîtres de la rue en 1936. C'était là qu'était tombé en 1914 un des obus du canon allemand Gross Bertha, et le café "La Vielleuse" en conservait fièrement les éclats, incrustés dans ses miroirs. Mais Belleville a subi dans les années soixante-dix un bombardement bien plus radical, presque un séisme. Les blocs résidentiels, les supermarchés, les rangées de bureaux ont évincé les petites rues et les anciennes maisons basses. Reconstruit à la moderne, le café "La Vielleuse" n'a conservé que son nom, et ses vieux habitués ont laissé place à des immigrés arabes ou noirs, chinois plus récemment, pour qui ni 1871, ni 1914, ni 1936 n'ont plus guère de signification. 14

L'évocation de la station Métro Opéra, vers la fin du roman (p. 186-187), est de la même veine.

À l'inverse, le Montréal patchwork,

- ville reprisée,

ville d'exils juxtaposés,

de solitudes amoncelées qui se côtoient sàns se voir (p. 186)

objet des séries du premier type, peut fort bien glisser dans la seconde catégorie, pour peu qu'il ait retrouvé une âme. Cette âme, on l'a vu, peut lui être conférée par sa dimension politique, ou plus généralement militante: les luttes politiques, syndicales, féministes ou culturelles restaurent la ville dans sa solidarité perdue. Elle naîtra également de l'avènement peut-être proche d'une société plurielle, appelée à remplacer un jour la ville schizophrène des premières séries. Cette société plurielle est mentionnée de plus en plus souvent vers la fin du roman et l'on ne sera pas surpris de constater qu'elle donne lieu à des séries du second type, qu'il s'agisse de l'évocation du marché Jean-Talon (p. 178-179) ou du rappel des pérégrinations de la narratrice à travers la ville (p. 198-199), par exemple. C'est d'ailleurs sur une énumération du second type que s'achève le livre, en' une vision réconciliatrice en définitive:

$\grave{A}$ nouveau, le Paris mouillé et gris de son adolescence, à nouveau le vacarme des machines à sous, les rumeurs anonymes au fond des bistros, les freins qui grincent au feu rouge, le reflet de la croix verte des pharmacies dans les flaques d'eau. À nouveau, les manifestations de la Nation à la 
République, à nouveau les défilés du premier mai. À nouveau... à propos, il paraît que la place du Québec est à SaintGermain-des-Prés. (p. 199-200)

Ainsi pour échapper à l'état de dé-solation - pour reprendre l'expression de Hannah Arendt ${ }^{15}$-, au déracinement, à la dérivation de sol qui guettent l'homme moderne, la seule issue possible est dans la solidarité: que celle-ci vienne du sentiment d'appartenance à une communauté (Mimi Yente et ses racines juives qu'elle transplante avec elle), de la volonté d'œuvrer à l'avènement d'une société plurielle qui remplacera la ville d'exils juxtaposés dont parle la narratrice, ou encore de la participation politique (du côté des luttes plutôt que de celui des partis). Est-il vraiment nécessaire de rappeler que les termes métropole et politique ont en commun l'étymon $\pi 0 \lambda \iota \sigma$ : le politique, c'est précisément - au départ en tout cas - de donner un sens à la cité; la métropole devient alors susceptible de redevenir une "ville-mère".

15 Citee par Jean Chesnaux, ibid., p. 172-173. 